Research Paper

\title{
5-Hydroxymethylcytosine and ten-eleven translocation dioxygenases in head and neck carcinoma
}

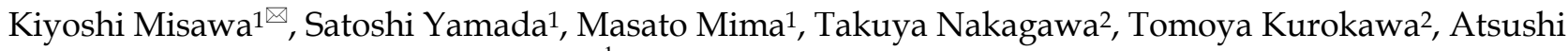
Imai ${ }^{1}$, Daiki Mochizuki ${ }^{1}$, Kotaro Morita ${ }^{1}$, Ryuji Ishikawa ${ }^{1}$, Shiori Endo ${ }^{1}$, and Yuki Misawa ${ }^{1}$

1. Department of Otolaryngology/Head and Neck Surgery, Hamamatsu University School of Medicine, Hamamatsu, Japan

2. Department of Otorhinolaryngology/Head and Neck Surgery, Graduate School of Medicine, Chiba University, Chiba, Japan

$\triangle$ Corresponding author: Kiyoshi Misawa, Department of Otolaryngology/Head and Neck Surgery, 1-20-1 Handayama, Hamamatsu University School of Medicine, Shizuoka, 431-3192, Japan; Phone: 81-53-435-2252, Fax: 81-53-435-2253; E-mail: kiyoshim@hama-med.ac.jp

(c) The author(s). This is an open access article distributed under the terms of the Creative Commons Attribution License (https://creativecommons.org/licenses/by/4.0/). See http://ivyspring.com/terms for full terms and conditions.

Received: 2019.03.12; Accepted: 2019.08.06; Published: 2019.08.28

\begin{abstract}
Ten-eleven translocation (TET) enzymes are implicated in DNA demethylation through dioxygenase activity, which converts 5-methylcytosine to 5-hydroxymethylcytosine $(5-\mathrm{hmC})$. However, the specific roles of TET enzymes and 5-hmC levels in head and neck squamous cell carcinoma (HNSCC) have not yet been evaluated. In this study, we analyzed 5-hmC levels and TET mRNA expression in a well-characterized dataset of 117 matched pairs of HNSCC tissues and normal tissues. 5-hmC levels and TET mRNA expression were examined via enzyme-linked immunosorbent assay and quantitative real-time PCR, respectively. 5-hmC levels were evaluated according to various clinical characteristics and prognostic implications. Notably, we found that 5 -hmC levels were significantly correlated with tumor stage $(P=0.032)$ and recurrence $(P=0.018)$. Univariate analysis revealed that low levels of 5 -hmC were correlated with poor disease-free survival (DFS; log-rank test, $P=0.038$ ). The expression of TET family genes was not associated with outcomes. In multivariate analysis, low levels of 5-hmC were evaluated as a significant independent prognostic factor of DFS (hazard ratio: $2.352,95 \%$ confidence interval: $1.136-4.896 ; P=0.021$ ). Taken together, our findings showed that reduction of TET family gene expression and subsequent low levels of $5-\mathrm{hmC}$ may affect the development of HNSCC.
\end{abstract}

Key words: 5-hmC, ELISA, TET, HNSCC, disease-free survival

\section{Introduction}

Head and neck squamous cell carcinomas (HNSCCs) are heterogeneous diseases that involve multiple sites and cellular origins within the upper aerodigestive tract [1]. Despite aggressive multimodal treatment, survival for patients with HNSCC remains poor. Nevertheless, some patients survive much longer than expected [2]. Therefore, identification of prognostic biomarkers as clinical or biological characteristics that provide information on the likely health outcomes of patients, irrespective of the treatment, is essential [3, 4]. In HNSCC, epigenetic inactivation associated with tumor-suppressor genes (TSGs) is more frequent than somatic mutations and may drive tumorigenic and progression potential [5-7]. Aberrant gene promoter methylation is a key event in cancer development and has attracted increasing interest in basic and translational oncology studies because of the induction of reversible chemical modifications $[8,9]$.

Enzymes of the ten-eleven translocation (TET) family catalyze the stepwise oxidation of 5-methylcytosine $(5-\mathrm{mC})$ in DNA to 5-hydroxymethylcytosine $(5-\mathrm{hmC})$ and further oxidation products, not only generating new epigenetic marks but also initiating active or passive demethylation pathways [10]. Although tissue- and cell type-specific variations occur, it has been estimated that approximately $5 \%$ of all cytosines in the genome of mammalian cells are marked as 5-mC, and less than $1 \%$ are marked as $5-\mathrm{hmC}$. Moreover, 5-formylcytosine (5-fC) and 5-carboxylcytosine (5-caC) are 10-1000-fold less abundant than 5-hmC $[11,12]$. Accordingly, 5-fC and 5-caC may simply be short-lived intermediates in the active demethylation 
process, whereas 5-hmC may be an active epigenetic mark that is stably maintained [13]. TET family proteins can convert 5-mC to $5-\mathrm{hmC}$, which is widely accepted as the sixth base in the mammalian genome, following 5-mC, the fifth base [14, 15]. The few clinical investigations that examined global DNA hydroxymethylation in relation to HNSCC have used genomic DNA from tumors.

Missense and truncating mutations in TET genes are present in nearly all solid tumor types at a relatively low frequency [16]. In the Cancer Genome Atlas cohort of HNSCC, TET1, TET2, and TET3 mutations were identified in nine $(1.8 \%)$, eight patients $(1.6 \%)$, and eight patients $(1.6 \%)$ of 510 patients, respectively [17]. Our report indicated that TET mRNA is downregulated in HNSCC owing to DNA methylation; this may be a critical event in HNSCC progression. In particular, TET3 methylation confers HNSCC with unique clinicopathological features [18].

Recent studies have shown that aberrant levels of TET genes and 5-hmC are associated with tumorigenesis in different types of cancers [19]. In a number of cancers, 5-hmC has been shown to be markedly decreased and associated with tumorigenesis, progression, and outcomes [20]. Simultaneous analyses of 5-hmC and TET genes are important for predicting tumorigenesis and biological behaviors and for the development of future targeted therapies for HNSCC. However, systematic studies of the epigenetic and transcriptional regulation of 5-hmC and TET genes in HNSCC are still needed.

Accordingly, in this study, we compared the 5-hmC profiles between normal mucosa and HNSCC tissues and characterized the associations between 5-hmC and HNSCC tumorigenesis, progression, and outcomes.

\section{Methods}

\section{Tumor samples}

In total, 117 primary HNSCC samples were obtained from patients during surgery at the Department of Otolaryngology, Hamamatsu University School of Medicine. All patients provided written informed consent, and the study protocol was approved by the Institutional Review Board of the Hamamatsu University School of Medicine (date of board approval: 2 October 2015, ethic code: 25-149). Clinical information, including age, sex, alcohol exposure, smoking status, tumor size, human papilloma virus (HPV) status, tumor size, lymph node status, stage, and recurrence, were obtained from the patients' clinical records.

\section{DNA extraction and ELISA for 5-hmC quantification}

The genomic DNA from 117 primary tumors and noncancerous mucosa was extracted using a QIAamp DNA Mini Kit (Qiagen, Hilden, Germany) according to the manufacturer's instructions. The $5 \mathrm{hmC}$ content of genomic DNA was determined with a Quest 5-hmC DNA ELISA Kit (Zymo Research, Irvine, CA, USA), according to the manufacturer's instructions. Assays were performed using $4 \mu \mathrm{g} / \mathrm{mL}$ anti-5-hmC polyclonal antibodies, loading $200 \mathrm{ng}$ of DNA per well. Absorbance at $430 \mathrm{~nm}$ was evaluated using a SynergyH1 microplate reader and Gen5 software (BioTek, Winooski, VT, USA). The amount of 5-hmC was calculated as a percentage based on a standard curve generated using kit controls.

\section{RNA extraction and $q R T-P C R$}

Total RNA was isolated using an RNeasy Plus Mini Kit (Qiagen), and cDNA was synthesized using a ReverTra Ace qPCR RT Kit (Toyobo, Tokyo, Japan). The mRNA levels of TET1, TET2, TET3, and glyceraldehyde 3-phosphate dehydrogenase $(G A P D H)$ were measured via qRT-PCR using SYBR Premix Ex Taq (Takara, Tokyo, Japan) and a Takara Thermal Cycler Dice Real Time System TP8000 (Takara). The data were analyzed using the $\Delta \Delta \mathrm{Ct}$ method. Primer sequences were as follows: TET1 forward (F), CCCTTGGAAATGCCATAGGAA; TET1 reverse (R), GAGAGCCTGCTGGAACTGTTG; TET2 F, GGCTGTTGGCCAGAGACTTA; TET2 R, ATACCTGTAGGTGTTTGCCTGTTTA; TET3 F, GCCAACTTCAACATACCCTGGAC; TET3 R, CACCTGGATGTGGGACTGTGTAA; GAPDH F, GCACCGTCAAGGCTGAGAAC; GAPDH R, TGGTGAAGACGCCAGTCTCTA.

\section{Data analysis and Statistics}

The 5-hmC and TET mRNA levels in tumors and normal mucosa and patient characteristics were analyzed statistically. Receiver-operator characteristic (ROC) curve analyses were performed for 5-hmC and TET mRNA levels and all patients for comparisons between tumor and normal tissues. DFS was measured from the date of the initial treatment to the date of diagnosis. Kaplan-Meier tests were used to calculate survival probabilities, and log-rank tests were used to compare survival rates. The prognostic value of methylation status was assessed by performing multivariate Cox proportional hazards analysis adjusting for age ( $\geq 65$ versus $<65$ years), sex, smoking status, alcohol intake, and tumor stage (I, II, and III versus IV). A p-value less than 0.05 was considered statistically significant. Statistical analyses were performed using StatMate IV software (ATMS 
Co. Ltd., Tokyo, Japan) and the Stata/SE 13.0 system (Stata Corporation, TX, USA).

\section{Results}

\section{Determination of 5-hmC levels by ELISA in HNSCCs and matched normal mucosa}

First, we examined the 5-hmC contents of DNA in 117 matched pairs of HNSCC and matched normal mucosa using ELISA. Cancer tissues had significantly

A
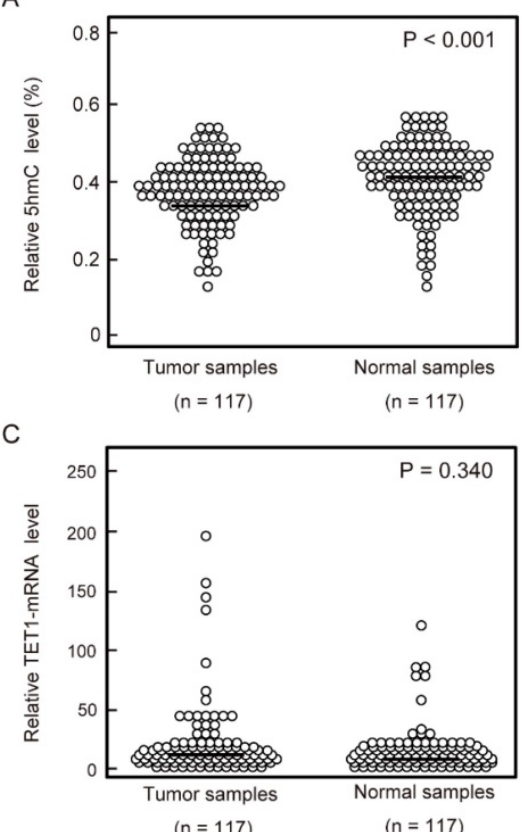

E

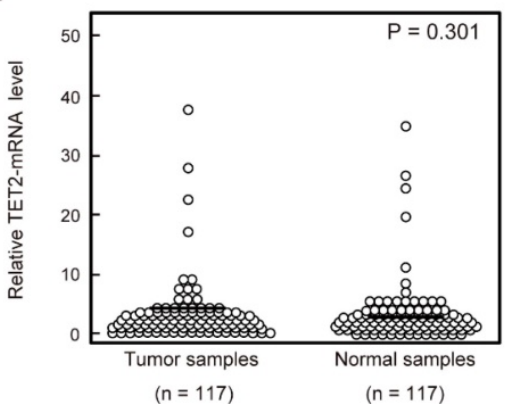

G

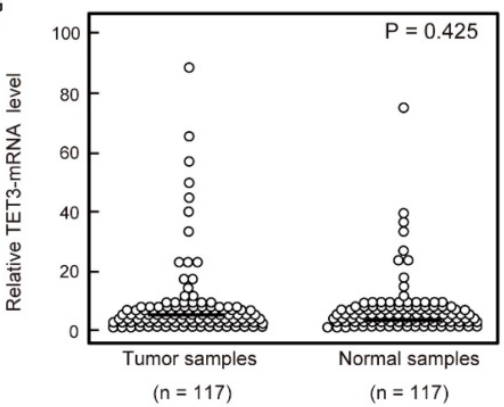

lower levels of $5-\mathrm{hmC}(0.373 \% \pm 0.087 \%)$ than matched normal mucosa $(0.406 \% \pm 0.090 \% ; P<0.001$ by paired t-tests). Notably, the 5 -hmC levels exhibited highly discriminative ROC curve profiles, which clearly distinguished HNSCC from normal mucosal tissues (area under the ROC [AUROC] = 0.612). At the cutoff value of 0.407 , the sensitivity and specificity were $57.3 \%$ and $64.1 \%$, respectively (Figure $1 \mathrm{~A}, 1 \mathrm{~B}$ ).

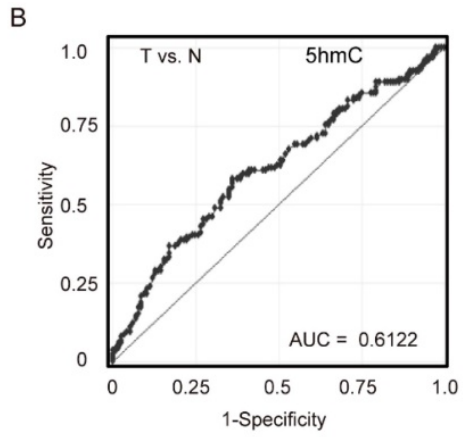

D

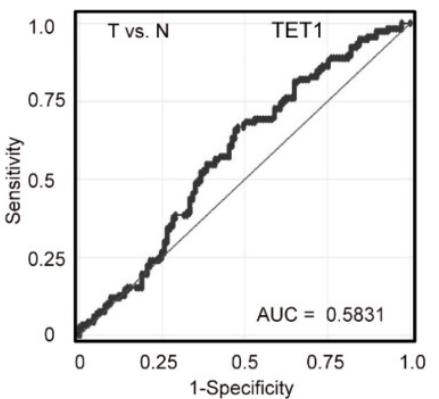

$\mathrm{F}$

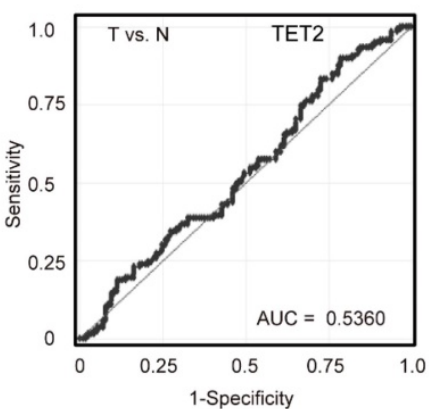

$\mathrm{H}$

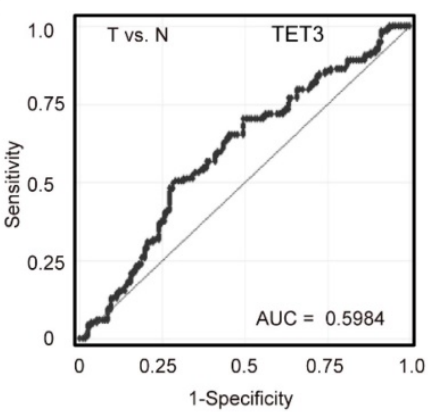

Figure 1. 5-hmC levels and TET mRNA patterns in matched pairs of HNSCC tissues and adjacent normal mucosal tissues. (A) ELISAs were used to determine the percentages of 5 -hmC levels in 117 matched pairs of HNSCC and normal mucosa specimens $(P<0.001)$. (B) The AUROC value for 5 -hmC was 0.6122 . At the cutoff value of 0.407 , the sensitivity was $57.3 \%$, and the specificity was $64.1 \%$. (C) Relative TET1 mRNA expression levels (P=0.340). (D) The AUROC value for TET1 was 0.583 . At the cutoff value of 0.580 , the sensitivity was $66.7 \%$, and the specificity was $52.1 \%$. (E) Relative TET2 mRNA expression levels $(P=0.301)$. (F) The AUROC value for TET2 was 0.536 . At the cutoff value of 0.1015 , the sensitivity was $82.9 \%$, and the specificity was $27.4 \%$. (G) Relative TET3 mRNA expression levels $(P=0.425)$. (H) The AUROC value for TET3 was 0.598 . At the cutoff value of 1.866 , the sensitivity was $50.4 \%$, and the specificity was $70.9 \%$. The significance of differences between cancerous and normal mucosal tissues were determined by Student's t-tests. **P $<0.001$. 
Table 1. Distribution of $5 \mathrm{hmC}$ level and TETs expression status by selected epidemiologic and clinical characteristics

\begin{tabular}{|c|c|c|c|c|c|c|c|c|c|c|c|}
\hline \multicolumn{3}{|l|}{ Characteristics } & \multicolumn{3}{|l|}{ Age } & \multicolumn{3}{|l|}{ Gender } & \multicolumn{3}{|c|}{ HPV status } \\
\hline Markers & Status & Overall (\%) & $<65$ & $>65$ & $P+$ & Female & Male & $\mathrm{P}+$ & positive & negative & $\mathrm{P}+$ \\
\hline \multirow[t]{2}{*}{$5 \mathrm{hmC}$ level } & High & $75(64.1 \%)$ & 20 & 22 & & 6 & 36 & & 8 & 34 & \\
\hline & Low & $42(35.9 \%)$ & 22 & 53 & 1 & 8 & 67 & 1 & 6 & 69 & 0.135 \\
\hline \multirow[t]{2}{*}{ TET1 expression } & High & $78(66.7 \%)$ & 27 & 51 & & 11 & 67 & & 11 & 67 & \\
\hline & Low & $39(33.3 \%)$ & 15 & 24 & 1 & 3 & 36 & 0.38 & 3 & 36 & 0.38 \\
\hline \multirow[t]{2}{*}{ TET2 expression } & High & $97(82.9 \%)$ & 34 & 63 & & 14 & 83 & & 13 & 84 & \\
\hline & Low & $20(17.1 \%)$ & 8 & 12 & 1 & 0 & 20 & 0.124 & 1 & 19 & 0.459 \\
\hline \multirow[t]{2}{*}{ TET3 expression } & High & $59(50.4 \%)$ & 22 & 37 & & 7 & 52 & & 7 & 52 & \\
\hline & Low & $58(49.6 \%)$ & 20 & 38 & 0.848 & 7 & 51 & 1 & 7 & 51 & 0.572 \\
\hline \multicolumn{2}{|l|}{ Characteristics } & \multicolumn{3}{|c|}{ Alcohol exposure } & \multicolumn{3}{|c|}{ Smoking status } & \multicolumn{4}{|c|}{ Tumor size } \\
\hline Markers & Status & drinker & non drinker & $P \dagger$ & smoker & non smoker & $\mathrm{P}+$ & $\mathrm{T} 1-2$ & T3-4 & $P \dagger$ & \\
\hline \multirow[t]{2}{*}{$5 \mathrm{hmC}$ level } & High & 36 & 6 & & 36 & 6 & & 15 & 27 & & \\
\hline & Low & 58 & 17 & 0.337 & 62 & 13 & 0.796 & 33 & 42 & 0.436 & \\
\hline \multirow[t]{2}{*}{ TET1 expression } & High & 61 & 17 & & 61 & 17 & & 33 & 45 & & \\
\hline & Low & 33 & 6 & 0.469 & 37 & 2 & $0.031^{*}$ & 15 & 24 & 0.842 & \\
\hline \multirow[t]{2}{*}{ TET2 expression } & High & 78 & 19 & & 78 & 19 & & 39 & 58 & & \\
\hline & Low & 16 & 4 & 1 & 20 & 0 & $0.040^{*}$ & 9 & 11 & 1 & \\
\hline \multirow[t]{2}{*}{ TET3 expression } & High & 49 & 10 & & 47 & 12 & & 27 & 32 & & \\
\hline & Low & 45 & 13 & 0.493 & 51 & 7 & 0.317 & 21 & 37 & 0.349 & \\
\hline \multicolumn{2}{|l|}{ Characteristics } & \multicolumn{3}{|c|}{ Lympho-node status } & \multicolumn{3}{|l|}{ Stage } & \multicolumn{4}{|c|}{ Recurrence events } \\
\hline Markers & Status & No & $\mathrm{N+}$ & $P+$ & I, II, III & IV & $\mathrm{P} \dagger$ & positive & negative & $P \dagger$ & \\
\hline \multirow[t]{2}{*}{$5 \mathrm{hmC}$ level } & High & 15 & 27 & & 11 & 31 & & 10 & 32 & & \\
\hline & Low & 31 & 44 & 0.693 & 35 & 40 & $0.032^{*}$ & 35 & 40 & $0.018^{*}$ & \\
\hline \multirow[t]{2}{*}{ TET1 expression } & High & 29 & 49 & & 31 & 47 & & 30 & 48 & & \\
\hline & Low & 17 & 22 & 1 & 15 & 24 & 1 & 15 & 24 & 1 & \\
\hline \multirow[t]{2}{*}{ TET2 expression } & High & 35 & 62 & & 36 & 61 & & 36 & 61 & & \\
\hline & Low & 11 & 9 & 0.136 & 10 & 10 & 1 & 9 & 11 & 1 & \\
\hline \multirow[t]{2}{*}{ TET3 expression } & High & 22 & 37 & & 25 & 34 & & 23 & 36 & & \\
\hline & Low & 24 & 34 & 0.707 & 21 & 37 & 0.571 & 22 & 36 & 1 & \\
\hline
\end{tabular}

† Chi-squared test

* $\mathrm{P}<0.05$

\section{TET expression in HNSCCs and matched normal mucosa}

Quantitative reverse transcription polymerase chain reaction (qRT-PCR) was performed to examine mRNA expression of TET1, TET2, and TET3 in 117 matched pairs of HNSCC and normal mucosa. There were no significant differences in TET1, TET2, and TET3 mRNA levels between cancerous and normal tissues (Figure 1C, 1E, 1G). TET1, TET2, and TET3 mRNA levels showed discriminative ROC curve profiles, which distinguished HNSCC from normal mucosal tissues $($ AUROC $=0.583,0.536$, and 0.598, respectively). Tumor samples were classified as positive when the mRNA expression levels exceeded $0.580,0.102$, and 1.866 for TET1, TET2, and TET3, respectively. The cutoff mRNA expression level was chosen from the ROC curve to maximize sensitivity and specificity (Figure 1D, 1F, 1H).

\section{Comparison of 5-hmC levels and TET expression in HNSCC tissues}

We found that 5 -hmC levels were significantly correlated with the relative mRNA levels of TET genes, including TET1 $\left(\mathrm{R}^{2}=0.0515, P=0.014\right)$, TET2 $\left(\mathrm{R}^{2}=0.052, P=0.013\right)$, and TET3 $\left(\mathrm{R}^{2}=0.064, P=0.006\right)$ (Figure 2A-C).

To identify factors affecting 5-hmC levels in HNSCC, we compared 5-hmC levels among the number of highly expressed TET genes and tumor sites of HNSCC. One or more TET high-expression events were associated with a significant increase in 5-hmC levels compared with no TET high-expression events $(P<0.05)$. The 5 -hmC level showed the greatest increase when all three TET genes showed high expression $(P<0.001$; Figure 3A). Moreover, in a comparison of 5-hmC levels at tumor sites among HNSCC, 5-hmC levels were found to be significantly higher in patients with oropharyngeal cancer than in patients with larynx $(P=0.019)$, oral cavity $(P=0.029)$, and hypopharynx $(P=0.009)$ cancer (Figure 3B).

\section{Association of 5-hmC levels and TET expression with clinicopathological assessment}

Among the 117 patients, differences in 5-hmC levels and TET1, TET2, and TET3 mRNA expression statuses according to clinical information were examined using Chi-squared tests (Table 1). The characteristics of patients with HNSCC are shown in Table S1. We found that 5 -hmC levels were associated with clinical stage $(P=0.032)$ and recurrence $(P=$ 0.018). Other clinical information, including age, sex, alcohol exposure, smoking status, tumor size, HPV status, tumor size, and lymph node status, was not related to 5-hmC levels. Smoking habit was associated with mRNA expression of TET1 $(P=0.031)$ and TET2 $(P=0.040)$. Other clinical information was not related to TET1, TET2, and TET3 mRNA expression (Table 1). Comparison of TET1, TET2, and TET3 mRNA 
expression in patients with laryngeal cancer, oral cancer, hypopharyngeal cancer, and oropharyngeal cancer are shown in Figure S1.

\section{5-hmC levels and TET expression in HNSCC and the relationship with patient survival}

Next, we confirmed the relationship between DFS in patients with HNSCC and 5-hmC levels/TET expression using Kaplan-Meier plots (Figure 4). Shorter DFS times were observed in patients with low 5-hmC levels compared with those with high 5-hmC levels (log-rank test, $P=0.038$; Figure $4 \mathrm{~A}$ ). There were

A

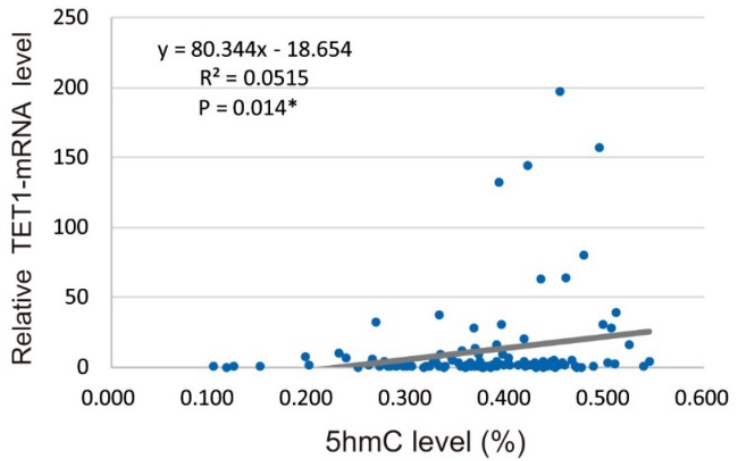

B

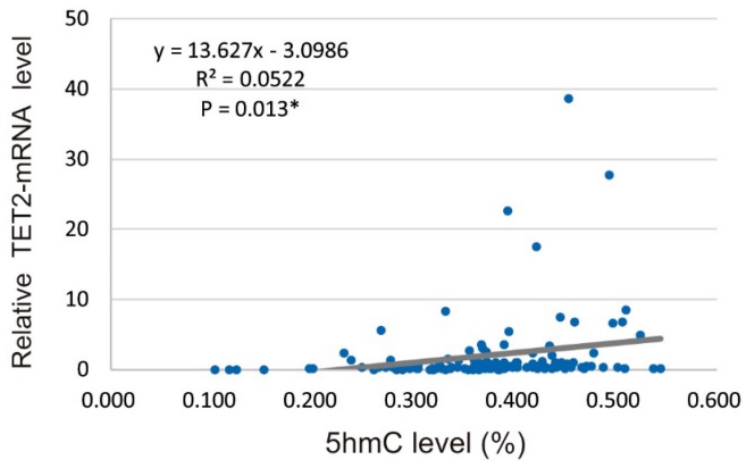

C

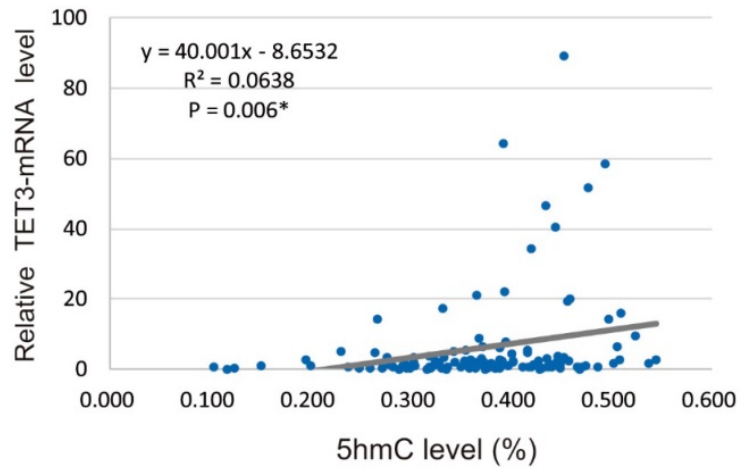

Figure 2. Spearman rank correlations between $5-\mathrm{hmC}$ and TET MRNA levels in 117 HNSCCs. (A) Correlation between 5-hmC levels and TET1 expression $\left(R^{2}=0.052, P=0.014\right)$. (B) Correlation between 5 -hmC levels and TET2 expression $\left(R^{2}=0.052, P=0.013\right)$. (C) Correlation between $5-\mathrm{hmC}$ levels and TET3 expression $\left(R^{2}=0.064, P=0.006\right)$ no relationships in DFS between the high and low expression groups for TET1 (78 versus 39, $P=0.955)$, TET2 (97 versus $20, P=0.479$ ), and TET3 ( 59 versus 58 , $P=0.887)$ among the 117 patients enrolled in this study (Figure 4B-D).

A

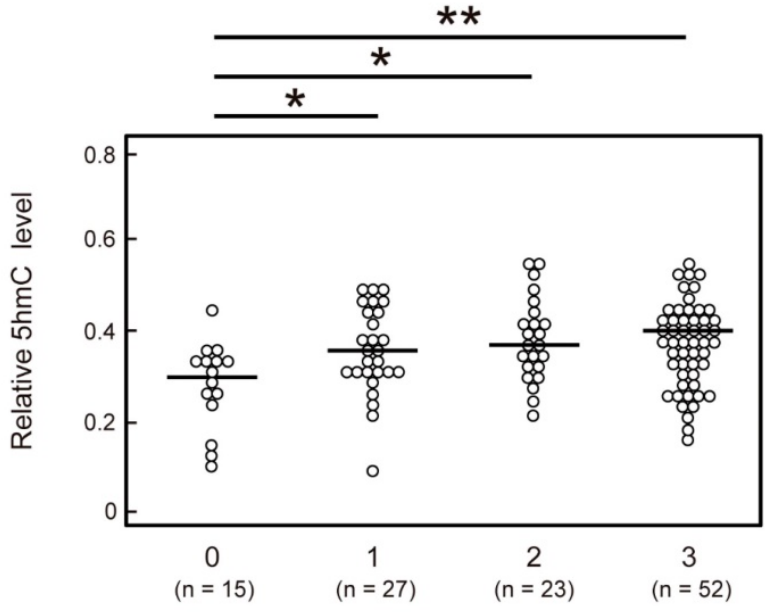

The number of TET high expression events

B

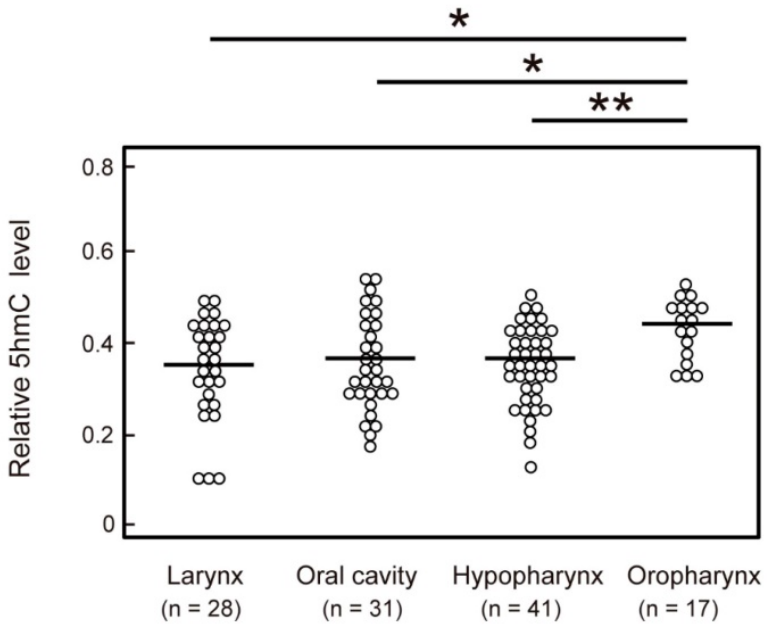

The tumor origin for four sites

Figure 3. Comparison of $5-\mathrm{hmC}$ levels and the number of TET high-expression events or the anatomical location of 117 HNSCCs. (A) Relationship between number of TET high-expression events and 5-hmC levels. 0: all TET genes low expression; 1: one TET genes high expression; 2 : two TET genes high expression; 3: all TET genes high expression. (B) Relationship between the anatomical location of the tumor and $5-\mathrm{hmC}$ levels. The significance of relationships between 5 -hmC levels and other factors was compared using Student's t-tests. *P $<0.05$; **P $<$ $0.01 ; * * * \mathrm{P}<0.001$.

Additionally, low 5-hmC levels were associated with decreased DFS compared with high 5-hmC levels in patients with $\mathrm{T} 3$ and $\mathrm{T} 4$ stages, positive lymph node metastasis, and stage IV disease $(P<$ $0.001, P=0.029, P<0.001$, and $P<0.001$, respectively; Figure 5B, 5D, 5F). 5-hmC levels in patients with T1 and T2 stages; negative lymph node metastasis; and 
stages I, II, and III tumors were not related to outcomes (Figure 5A, 5C, 5E).

The associations of the risk of recurrence with 5-hmC levels and TET1, TET2, and TET3 statuses were estimated using multivariate analysis with Cox proportional hazards models adjusted for age, sex, smoking status, alcohol exposure, and stage. In patients with high 5-hmC levels (64.1\%), the adjusted risk ratio for recurrence (RR) was $2.352 \quad(95 \%$ confidence interval [CI]: 1.136-4.869, $P=0.021$ ). 5 -hmC levels correlated positively with recurrence in patients with $\mathrm{T} 3$ and $\mathrm{T} 4$ tumor stages and positive lymph node metastasis (RR, 4.33; 95\% CI, 1.62-11.5; $P$ $=0.003$ and $\mathrm{RR}, 3.38 ; 95 \% \mathrm{CI}, 1.18-9.65 ; P=0.023$, respectively; Figure 6).

\section{Discussion}

This is the first study examining $5-\mathrm{hmC}$ and TET family gene levels in HNSCC. DNA methylation

A

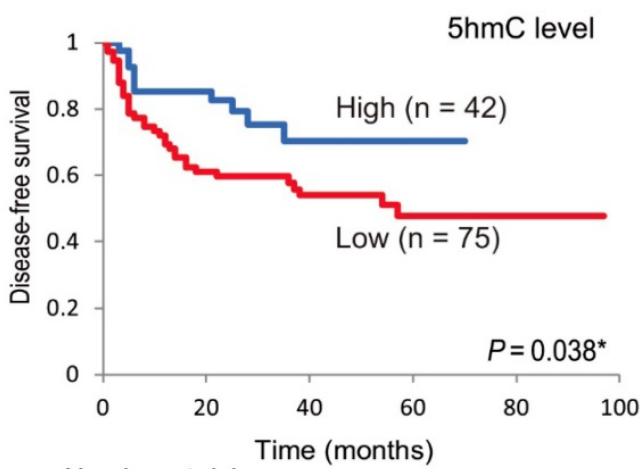

Number at risk

\begin{tabular}{|c|c|c|c|c|c|}
\hline Time (Months) & 0 & 20 & 40 & 60 & 80 \\
\hline Low & 75 & 44 & 28 & 10 & 2 \\
\hline High & 42 & 31 & 13 & 6 & 0 \\
\hline
\end{tabular}

C

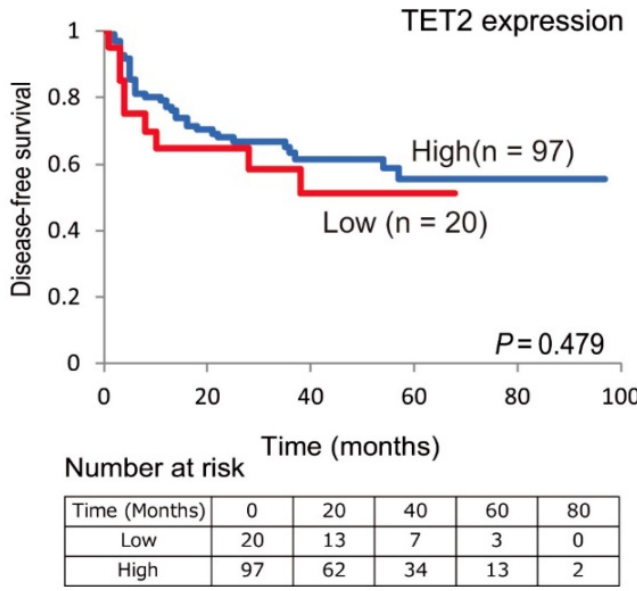

regulates epigenetic gene inactivation; however, the factors affecting DNA demethylation are still poorly understood in HNSCC. Recently, we showed that concurrent methylation analysis of TET genes was related to reduced DFS in unfavorable event groups [18]. Our current study found that aberrant expression of TET genes and altered levels of $5-\mathrm{hmC}$ were associated with tumorigenesis and that lower 5-hmC levels were correlated with reduced survival.

Loss of 5-hmC is associated with decreased expression of TET1 and TET2 in small intestinal neuroendocrine tumors [21]. Moreover, 5-hmC levels are significantly reduced in prostate cancer compared with normal prostate tissue samples [22]. In esophageal cancer tissues, 5-hmC expression is associated with shorter overall survival and TET2 expression levels [23]. TET proteins catalyze DNA $\mathrm{CpG}$ demethylation through converting 5-mC to 5-hmC, maintaining a delicate balance between $\mathrm{CpG}$

B

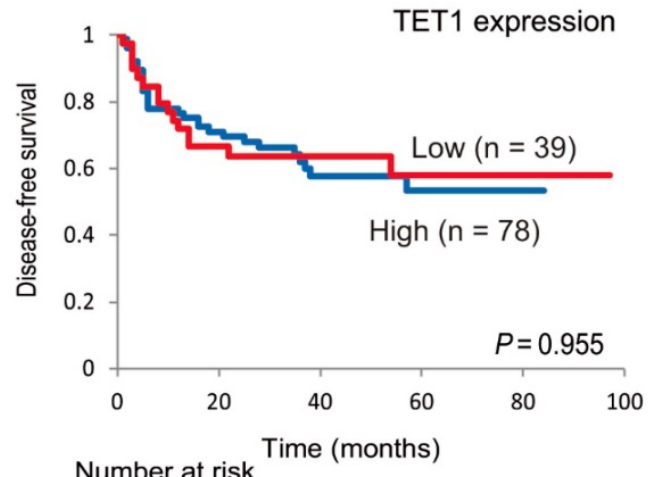

\begin{tabular}{|c|c|c|c|c|c|}
\hline Time (Months) & 0 & 20 & 40 & 60 & 80 \\
\hline Low & 39 & 25 & 15 & 8 & 1 \\
\hline High & 78 & 50 & 26 & 8 & 1 \\
\hline
\end{tabular}

D

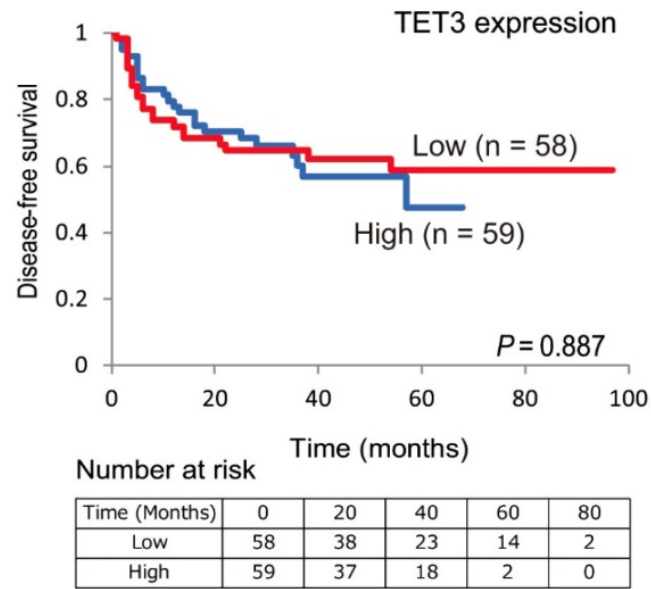

Figure 4. Kaplan-Meier survival curves based on 5-hmC levels and TET expression status in patients with HNSCC. DFS according to (A) 5-hmC levels; (B) TETI expression status; (C) TET2 expression status; (D) TET3 expression status. 
methylation and demethylation in normal cells [24]. Notably, promoter CpG methylation-mediated silencing of the TET1 gene further increases $5-\mathrm{mC}$

A

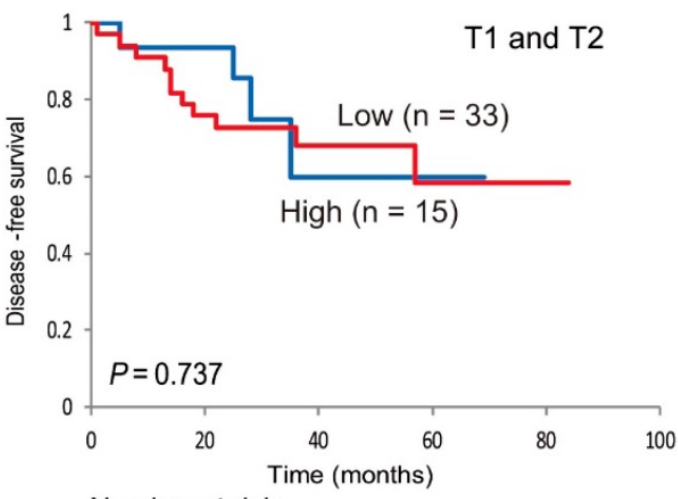

Number at risk

\begin{tabular}{|c|c|c|c|c|c|}
\hline Time (Months) & 0 & 20 & 40 & 60 & 80 \\
\hline Low & 33 & 25 & 15 & 6 & 1 \\
\hline High & 15 & 14 & 3 & 3 & 0 \\
\hline
\end{tabular}

C

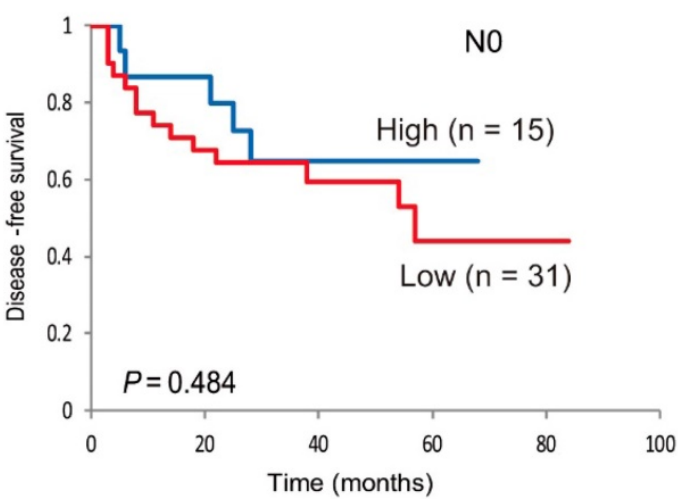

Number at risk

\begin{tabular}{|c|c|c|c|c|c|}
\hline Time (Months) & 0 & 20 & 40 & 60 & 80 \\
\hline Low & 31 & 21 & 12 & 3 & 1 \\
\hline High & 15 & 13 & 7 & 2 & 0 \\
\hline
\end{tabular}

E

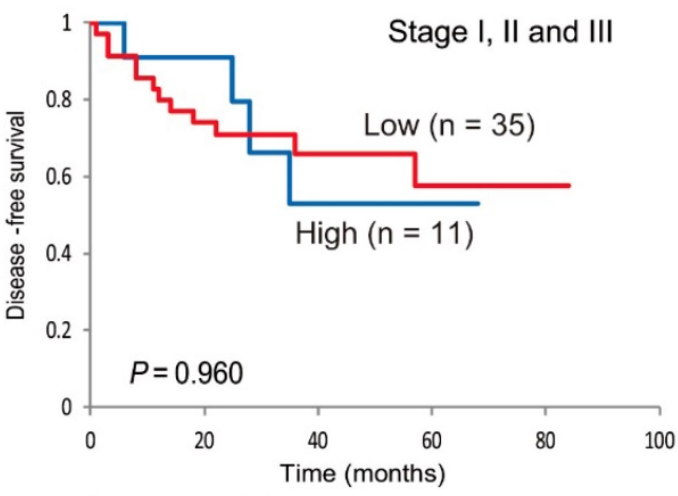

Number at risk

\begin{tabular}{|c|c|c|c|c|c|}
\hline Time (Months) & 0 & 20 & 40 & 60 & 80 \\
\hline Low & 35 & 24 & 14 & 6 & 1 \\
\hline High & 11 & 9 & 4 & 2 & 0 \\
\hline
\end{tabular}

levels in tumor cells, thus forming a DNA methylation feedback loop mediated by DNMT and TET1 [25].

B

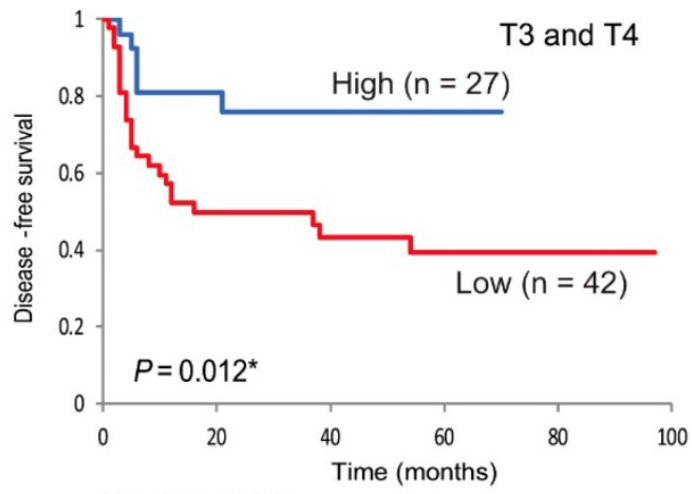

Number at risk

\begin{tabular}{|c|c|c|c|c|c|}
\hline Time (Months) & 0 & 20 & 40 & 60 & 80 \\
\hline Low & 42 & 19 & 13 & 4 & 1 \\
\hline High & 27 & 17 & 10 & 3 & 0 \\
\hline
\end{tabular}

D

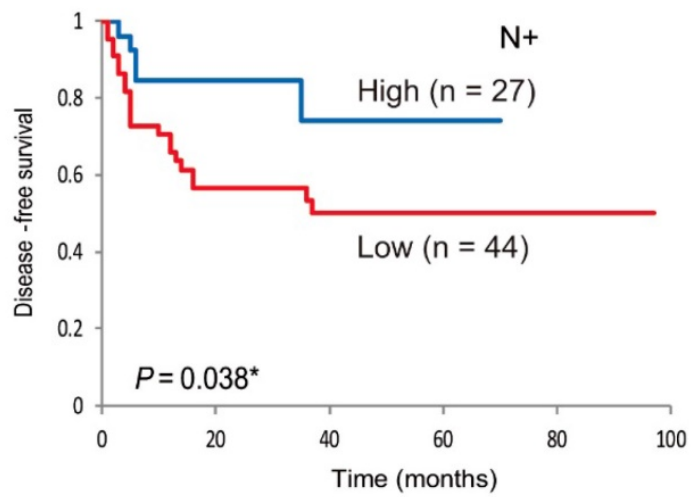

Number at risk

\begin{tabular}{|c|c|c|c|c|c|}
\hline Time (Months) & 0 & 20 & 40 & 60 & 80 \\
\hline Low & 44 & 23 & 16 & 7 & 1 \\
\hline High & 27 & 18 & 6 & 4 & 0 \\
\hline
\end{tabular}

$\mathrm{F}$

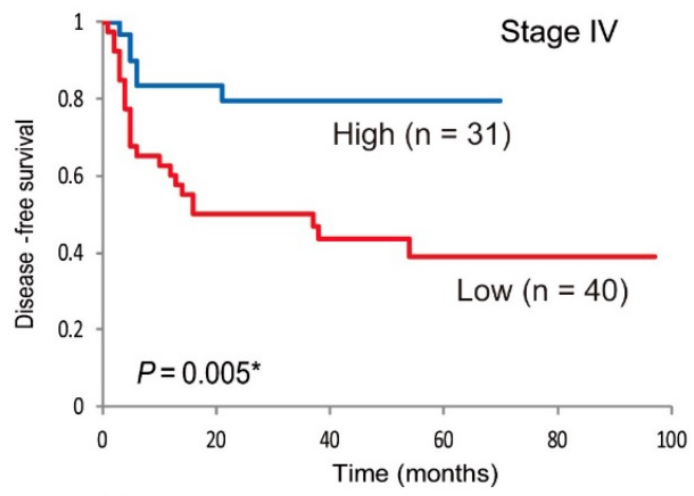

Number at risk

\begin{tabular}{|c|c|c|c|c|c|}
\hline Time (Months) & 0 & 20 & 40 & 60 & 80 \\
\hline Low & 40 & 20 & 14 & 4 & 1 \\
\hline High & 31 & 22 & 9 & 4 & 0 \\
\hline
\end{tabular}

Figure 5. Kaplan-Meier survival curves based on $\mathbf{5}-\mathbf{h m C}$ levels in patients with HNSCC. DFS for $(A)$ tumor size in $\mathrm{T} 1$ and $\mathrm{T} 2$ cases $(\mathrm{n}=48)$; $(\mathrm{B})$ tumor size in $\mathrm{T} 3$ and T4 cases ( $n=69)$; (C) lymph node status in N0 cases $(n=46)$; (D) lymph node status in N+ cases $(n=71)$; $(E)$ stage I, II, and III cases $(n=46)$; and $(F)$ stage IV cases $(n=71)$. 


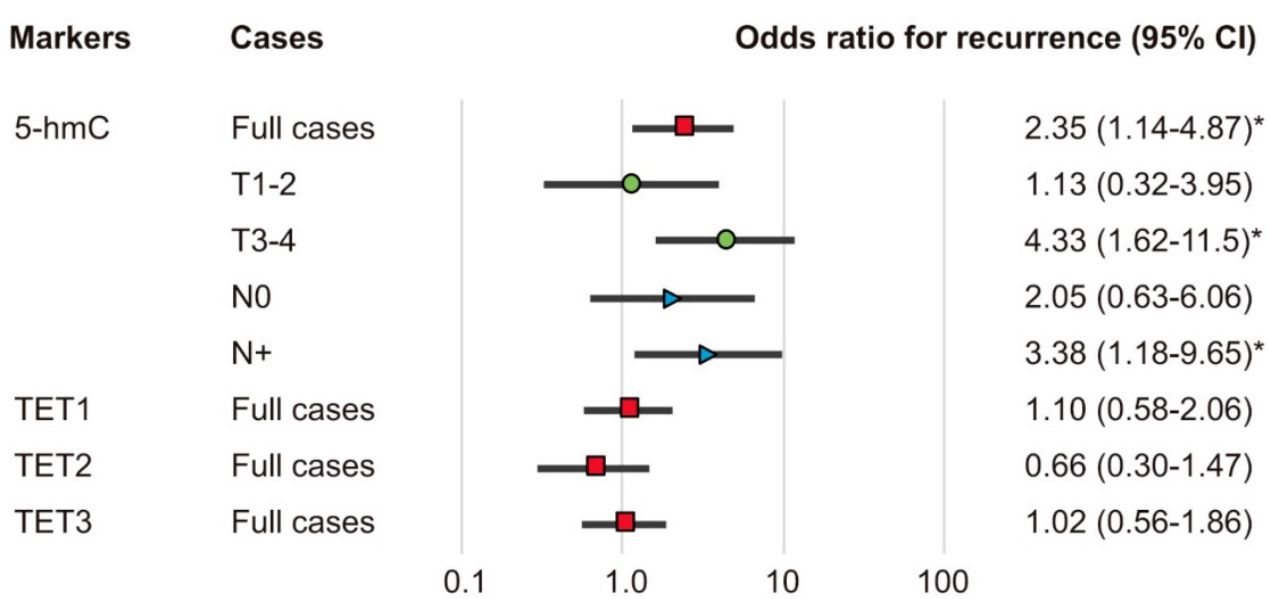

Figure 6. Odds ratios for recurrence based on Cox proportional hazards models. Cox proportional hazards model, revealing the estimated odds of recurrence associated with 5-hmC levels and TET1, TET2, and TET3 expression; Cl: confidence interval. *P $<0.05$.

5-hmC is not simply an activating epigenetic mark, but is considered an intermediate in the active demethylation pathway and appears to play complex roles in gene regulation [11, 12]. 5-hmC levels of protein-coding genes are positively correlated with RNA expression intensity [26]. A pathway recently suggested for active DNA demethylation in the early mouse embryo involves the conversion of 5-mC to 5 -hmC mediated by TET3, which is expressed at high levels in oocytes and zygotes [27, 28]. Future studies are needed to confirm the associations between 5-hmC and carcinogenesis and to examine potential mechanisms through which 5-hmC loss affects tumor growth.

Bisulfite treatment, the gold-standard technology for detection of DNA methylation, results in the conversion of unmethylated cytosine into uracil, which will be read as thymine after PCR amplification, with both $5-\mathrm{mC}$ and $5-\mathrm{hmC}$ being read as cytosine, cannot distinguish between 5-mC and 5-hmC [29]. Therefore, quantitative analysis of genome-wide distribution of these epigenetic marks has been considered for clinical applications [30]. Immuno-based assays, including dot blots, immunohistochemical assays, and ELISA, have widely been used as a quantitative method due to their analytical merits for analyses of 5-hmC [31]. Several approaches for 5-hmC mapping have been developed in recent years. Cell-free $5-\mathrm{hmC}$ may represent a new approach for liquid biopsy-based diagnosis and prognosis [32, 33]. The $5-\mathrm{hmC}$ profiles of cell-free DNA have been detected in patients with cancer, and 5-hmC gains in both gene bodies and promoter regions have been evaluated in patients with cancer and healthy controls [34]. Further studies of the loss of 5-hmC upon transformation of tissues may offer useful tools for dissecting 5-hmC biology in cancers.
In summary, we demonstrated for the first time that 5 -hmC levels were abnormally reduced in patients with HNSCC; this may be a critical event in HNSCC progression. Interestingly, the 5 -hmC profiles in primary tumors may be used to identify patients with positive lymph node metastasis and high tumor stage that are at a higher risk of recurrence. Further studies are needed to examine the differences in global demethylation patterns observed between 5-hmC-low and -high tumors and their effects on the onset and progression of HNSCC in more detail.

\section{Supplementary Material}

Supplementary figure and table. http://www.jcancer.org/v10p5306s1.pdf

\section{Acknowledgments}

The authors would like to thank Ms. Yuko Mohri for her excellent technical support.

\section{Financial disclosure}

This study was funded by a Grant-in-Aid for Scientific Research (No. 16K11228, No. 16K20239, No. 17K11380, No. 17K16903 and No. 17K16904) from the Ministry of Education, Culture, Sports, Science, and Technology of Japan.

\section{Ethical disclosure}

All samples were obtained with the written consent of patients. The study was approved by the ethical committee of Hamamatsu University School of Medicine (number 16-072).

\section{Author Contributions}

K Misawa designed the experiments. Y Misawa and $\mathrm{K}$ Misawa conducted all experiments. Y Misawa and $\mathrm{K}$ Misawa wrote the manuscript. S Yamada, M Mima, T Nakagawa, T Kurokawa, A Imai, D Mochizuki, K Morita, R Ishikawa, S Endo and Y 
Misawa performed the data analysis and discussed the results. All authors read and approved the final manuscript.

\section{Competing Interests}

The authors have declared that no competing interest exists.

\section{References}

1. Pai SI, Westra WH. Molecular pathology of head and neck cancer: implications for diagnosis, prognosis, and treatment. Annual review of pathology. 2009; 4: 49-70.

2. Bose P, Brockton NT, Dort JC. Head and neck cancer: from anatomy to biology. International journal of cancer. 2013; 133: 2013-23.

3. Kang H, Kiess A, Chung CH. Emerging biomarkers in head and neck cancer in the era of genomics. Nature reviews Clinical oncology. 2015; 12: 11-26.

4. Mochizuki D, Adams A, Warner KA, Zhang Z, Pearson AT, Misawa K, et al. Anti-tumor effect of inhibition of IL-6 signaling in mucoepidermoid carcinoma. Oncotarget. 2015; 6: 22822-35.

5. Stransky N, Egloff AM, Tward AD, Kostic AD, Cibulskis K, Sivachenko A, et al. The mutational landscape of head and neck squamous cell carcinoma. Science. 2011; 333: 1157-60.

6. Kanazawa T, Misawa K, Misawa Y, Uehara T, Fukushima H, Kusaka G, et al. G-Protein-Coupled Receptors: Next Generation Therapeutic Targets in Head and Neck Cancer? Toxins. 2015; 7: 2959-84.

7. Misawa K, Misawa Y, Kanazawa T, Mochizuki D, Imai A, Endo S, et al. Epigenetic inactivation of galanin and GALR1/2 is associated with early recurrence in head and neck cancer. Clinical \& experimental metastasis. 2016; 33: 187-95.

8. Laytragoon-Lewin N, Rutqvist LE, Lewin F. DNA methylation in tumour and normal mucosal tissue of head and neck squamous cell carcinoma (HNSCC) patients: new diagnostic approaches and treatment. Med Oncol. 2013; 30: 654 .

9. Koffler J, Sharma S, Hess J. Predictive value of epigenetic alterations in head and neck squamous cell carcinoma. Molecular \& cellular oncology. 2014; 1: e954827.

10. An J, Rao A, Ko M. TET family dioxygenases and DNA demethylation in stem cells and cancers. Experimental \& molecular medicine. 2017; 49: e323.

11. Ito S, Shen L, Dai Q, Wu SC, Collins LB, Swenberg JA, et al. Tet proteins can convert 5-methylcytosine to 5-formylcytosine and 5-carboxylcytosine. Science. 2011; 333: 1300-3.

12. Song CX, Yi C, He C. Mapping recently identified nucleotide variants in the genome and transcriptome. Nature biotechnology. 2012; 30: 1107-16.

13. Shen L, Song CX, He C, Zhang Y. Mechanism and function of oxidative reversal of DNA and RNA methylation. Annual review of biochemistry. 2014; 83: 585-614.

14. Ye C, Li L. 5-hydroxymethylcytosine: a new insight into epigenetics in cancer. Cancer biology \& therapy. 2014; 15: 10-5.

15. Wu H, Zhang Y. Mechanisms and functions of Tet protein-mediated 5-methylcytosine oxidation. Genes \& development. 2011; 25: 2436-52.

16. Rasmussen KD, Helin K. Role of TET enzymes in DNA methylation, development, and cancer. Genes \& development. 2016; 30: 733-50.

17. Cancer Genome Atlas Network. Comprehensive genomic characterization of head and neck squamous cell carcinomas. Nature 2015 : 517: 576-82.

18. Misawa K, Imai A, Mochizuki D, Mima M, Endo S, Misawa Y, et al. Association of TET3 epigenetic inactivation with head and neck cancer. Oncotarget. 2018; 9: 24480-93.

19. Pfeifer GP, Xiong W, Hahn MA, Jin SG. The role of 5-hydroxymethylcytosine in human cancer. Cell and tissue research. 2014; 356: 631-41.

20. Johnson KC, Houseman EA, King JE, von Herrmann KM, Fadul CE, Christensen BC. 5-Hydroxymethylcytosine localizes to enhancer elements and is associated with survival in glioblastoma patients. Nature communications. 2016; 7: 13177

21. Barazeghi $E$, Prabhawa $S$, Norlen $O$, Hellman P, Stalberg P, Westin G Decrease of 5-hydroxymethylcytosine and TET1 with nuclear exclusion of TET2 in small intestinal neuroendocrine tumors. BMC cancer. 2018; 18: 764

22. Storebjerg TM, Strand SH, Hoyer S, Lynnerup AS, Borre M, Orntoft TF, et al. Dysregulation and prognostic potential of 5-methylcytosine $(5 \mathrm{mC})$, 5-hydroxymethylcytosine (5hmC), 5-formylcytosine (5fC), and 5 -carboxylcytosine $(5 \mathrm{caC})$ levels in prostate cancer. Clinical epigenetics. 2018; 10: 105.

23. Murata A, Baba Y, Ishimoto T, Miyake K, Kosumi K, Harada K, et al. TET family proteins and 5-hydroxymethylcytosine in esophageal squamous cell carcinoma. Oncotarget. 2015; 6: 23372-82.

24. Scourzic L, Mouly E, Bernard OA. TET proteins and the control of cytosine demethylation in cancer. Genome medicine. 2015; 7: 9.

25. Li L, Li C, Mao H, Du Z, Chan WY, Murray P, et al. Epigenetic inactivation of the CpG demethylase TET1 as a DNA methylation feedback loop in human cancers. Scientific reports. 2016; 6: 26591
26. Lin IH, Chen YF, Hsu MT. Correlated 5-Hydroxymethylcytosine (5hmC) and Gene Expression Profiles Underpin Gene and Organ-Specific Epigenetic Regulation in Adult Mouse Brain and Liver. PloS one. 2017; 12: e0170779.

27. Iqbal K, Jin SG, Pfeifer GP, Szabo PE. Reprogramming of the paternal genome upon fertilization involves genome-wide oxidation of 5-methylcytosine. Proceedings of the National Academy of Sciences of the United States of America. 2011; 108: 3642-7.

28. Peat JR, Dean W, Clark SJ, Krueger F, Smallwood SA, Ficz G, et al. Genome-wide bisulfite sequencing in zygotes identifies demethylation targets and maps the contribution of TET3 oxidation. Cell reports. 2014; 9: 1990-2000.

29. Skvortsova K, Zotenko E, Luu PL, Gould CM, Nair SS, Clark SI, et al. Comprehensive evaluation of genome-wide 5-hydroxymethylcytosine profiling approaches in human DNA. Epigenetics \& chromatin. 2017; 10: 16.

30. Chowdhury $\mathrm{B}$, Cho $\mathrm{IH}$, Hahn $\mathrm{N}$, Irudayaraj J. Quantification of 5-methylcytosine, 5-hydroxymethylcytosine and 5-carboxylcytosine from the blood of cancer patients by an enzyme-based immunoassay. Analytica chimica acta. 2014; 852: 212-7.

31. Gilat N, Tabachnik T, Shwartz A, Shahal T, Torchinsky D, Michaeli Y, et al. Single-molecule quantification of 5-hydroxymethylcytosine for diagnosis of blood and colon cancers. Clinical epigenetics. 2017; 9: 70.

32. Li W, Zhang X, Lu X, You L, Song Y, Luo Z, et al. 5-Hydroxymethylcytosine signatures in circulating cell-free DNA as diagnostic biomarkers for human cancers. Cell research. 2017; 27: 1243-57.

33. Song $\mathrm{CX}$, Yin $\mathrm{S}$, Ma $\mathrm{L}$, Wheeler $\mathrm{A}$, Chen $\mathrm{Y}$, Zhang $\mathrm{Y}$, et al 5-Hydroxymethylcytosine signatures in cell-free DNA provide information about tumor types and stages. Cell research. 2017; 27: 1231-42.

34. Zhang J, Han X, Gao C, Xing Y, Qi Z, Liu R, et al. 5-Hydroxymethylome in Circulating Cell-free DNA as A Potential Biomarker for Non-small-cell Lung Cancer. Genomics, proteomics \& bioinformatics. 2018; 16: 187-99. 